\title{
Esporotricose em crianças e adolescentes atendidos no HUPE-UERJ entre 1997 e 2010: estudo clinicoepidemiológico
}

\author{
Sporotrichosis in children and teenagers at HUPE-UERJ \\ between 1997-2010: a clinicoepidemiological study
}

\author{
Esporotricosis en niños y adolescentes atendidos en el HUPE-UERJ \\ entre 1997 y 2010: estudio clinico-epidemiológico
}

\author{
Andréa R. Bernardes-Engemann, 'Leila M. Lopes-Bezerra, \\ Priscila M. de Macedo, Rosane Orofino-Costa
}

\begin{abstract}
Resumo
Desde 1997, o Rio de Janeiro enfrenta uma epidemia de transmissão zoonótica de esporotricose, sendo a população de crianças e adolescentes comumente afetada pelo contato próximo com gatos doentes ou portadores do fungo. A pele é o local geralmente afetado com manifestações clínicas diversas. O tratamento de escolha é o itraconazol, mas iodeto de potássio e terbinafina são opções terapêuticas valiosas entre as crianças. O objetivo do presente artigo é estudar as características clinicoepidemiológicas de crianças e adolescentes com esporotricose atendidos no HUPE-UERJ. Foram incluídos pacientes com esporotricose, sem preferência por sexo ou etnia, faixa etária compreendida entre 0 e 18 anos. Dados como forma de contágio, forma clínica, localização das lesões e títulos sorológicos foram avaliados. A maioria dos 37 pacientes estudados encontrava-se na faixa etária entre 6 e 15 anos de idade, sem diferenças estatísticas quanto às variáveis sexo e cor. O contato com o gato foi a principal forma de contágio, e a forma linfocutânea predominou. O iodeto de potássio foi a droga mais utilizada, sendo o tempo médio de tratamento de 60 dias. Houve correlação clinicossorológica.
\end{abstract}

Descritores: Sporothrix; lodeto de potássio; Esporotricose.

\begin{abstract}
Since 1997, the city of Rio de Janeiro, Brazil, faces a zoonotic epidemics of sporotrichosis. Children and adolescents are particularly affected due to close contact with sick or asymptomatic carrier cats. The skin is commonly affected with many clinical presentations. Itraconazole is considered the treatment of choice; however, potassium iodide and terbinafine are valuable therapeutic options in children. To evaluate clinical and epidemiological profile of children and adolescents affected by sporotrichosis and treated at HUPE-UERJ. Children and adolescents between 0-18 years old diagnosed with sporotrichosis were included, regardless of gender or ethnicity. Data as type of transmission, clinical presentation, affected regions and serology titers was evaluated. Most of the 37 patients were in the age group between 6 and 15 years old, with no statistical differences in terms of sex and race. The main type of transmission was through contact with cats, and the lymphocutaneous form prevailed. Potassium iodide was the most prescribed drug, and the average treatment duration was 60 days. Study results showed clinical and serology correlation
\end{abstract}

Keywords: Sporothrix; Potassium iodide; Sporotrichosis.

\section{Resumen}

Desde 1997, Rio de Janeiro enfrenta una epidemia de transmisión zoonótica de esporotricosis, siendo la población de niños y adolescentes comúnmente afectada por el contacto cercano con gatos enfermos o portadores de hongo. La piel es el lugar comúnmente afectado con manifestaciones clínicas diversas. El tratamiento elegido es el itraconazol, pero el yodato de potasio y la terbinafina son opciones terapeúticas valiosas entre los niños. El objetivo del presente artículo es estudiar las características clinico-epidemiológicas de los niños y adolescentes con esporotricosis 
atendidos en el HUPE-UERJ. Fueron incluídos niños y adolescentes con esporotricosis, sin preferencia por el sexo o la etnia, grupo etáreo entre zero y 18 años. Datos como la forma de contagio, forma clínica, localización de las lesiones y títulos serológicos fueron evaluados. La mayoría de los 37 pacientes estudiados se encontraba en el grupo etáreo entre 6 y 15 años de edad, sin diferencias estadísticas en cuanto a las variables de sexo y color. El contacto con el gato fue la principal forma de contagio, y la forma linfocutánea predomino. El yodato de potasio fue la droga más utilizada, siendo el tiempo medio de tratamiento de 60 días. Hubo correlación clínica serológica.

Palabras clave: Sporothrix; Yoduro de potasio; Esporotricosis.

\section{Introdução}

A esporotricose é uma doença infecciosa causada pela inoculação traumática de fungos do complexo Sporothrix schenckii no hospedeiro humano (Figura 1). Desde 1997, no estado do Rio de Janeiro, observa-se uma epidemia da doença de transmissão zoonótica felina, predominantemente na capital e nos municípios da Baixada Fluminense. ${ }^{1,2}$ No contexto desta epidemia, as crianças são bastante afetadas por brincarem com os animais nas regiões peridomiciliares. ${ }^{3}$ As formas clínicas mais comuns limitam-se à pele e ao tecido linfático nos indivíduos imunocompetentes, como, por exemplo, a linfocutânea e cutânea fixa (Figura 2).,2,3 O atraso no diagnóstico e o uso inadvertido de múltiplos esquemas antimicrobianos levam à progressão da doença, causando dor e, posteriormente, cicatrizes inestéticas. Na América Latina, o tratamento de escolha para as formas cutâneas da doença é feito com a solução saturada de iodeto de potássio (SSKI), por sua eficácia conhecida e seu custo acessível. $^{4,5}$ Este medicamento é especialmente vantajoso para o uso em crianças por sua apresentação líquida; essa faixa etária, habitualmente, não tem contraindicações. Segundo orientações de guia de conduta terapêutica internacional, a dose tradicional preconizada para crianças é, inicialmente, de uma gota três vezes ao dia, com incrementos diários de uma gota a cada tomada, até que se atinja a dose máxima de uma gota/kg (máximo de 40 a 50 gotas) da solução concentrada $1 \mathrm{~g} / \mathrm{ml}$ ( 1 gota $=0,05 \mathrm{~g}$ ), que corresponde ao máximo de 6 a 7,5 g/dia. ${ }^{6} \mathrm{O}$ objetivo desse estudo foi examinar as características epidemiológicas, clínicas e terapêuticas das crianças e adolescentes com esporotricose atendidos no Serviço de Dermatologia e Laboratório de Micologia do Hospital Universitário Pedro Ernesto (HUPE) da Universidade do Estado do Rio de Janeiro (UERJ).

\section{Casuística e métodos}

Pacientes: foram selecionados 37 pacientes, entre zero e 18 anos, diagnosticados no período de setembro de 1997 a agosto de 2010 . As características clínicas e os aspectos epidemiológicos desses pacientes, bem como a evolução terapêutica, foram avaliados.

Métodos: a confirmação da esporotricose foi feita pelo diagnóstico clínico, micológico e/ou sorológico (por meiocda técnica de ELISA, usando a fração antigênica SsCBF). ${ }^{7}$ Todos os pacientes tiveram avaliação clinicossorológica antes do tratamento e acompanhamento mensal durante o tratamento até a cura clínica. Após o término do tratamento, essa avaliação ocorria a cada 3 meses até a negativação sorológica ou até completar 12 meses, o que ocorresse primeiro. O critério de cura foi determinado pela avaliação clínica.

\section{Resultados}

Dos 37 pacientes avaliados, a maioria encontrava-se na faixa etária entre 6 e 15 anos, morava na capital, seguida dos municípios da Baixada Fluminense. Não foram observadas diferenças estatísticas quanto às variáveis sexo e cor. O contato com o gato foi o principal meio de contágio (Figura 3). A forma linfocutânea predominou, e os locais mais afetados foram os membros superiores (72\%), seguidos pela face (52\%) (Figura 4). O tratamento administrado era preferencialmente o iodeto de potássio (KI), sob a forma de solução saturada (SSKI - 1,42 g/ml, em que uma gota equivale a $0,07 \mathrm{~g}) .{ }^{8}$ Essa medicação mostrou ter bom perfil de tolerância e não ocorreram eventos adversos graves, sendo o tempo médio de tratamento de 60 dias. A dose diária da SSKI variou entre 1,4 a 5,25 g. Dois pacientes utilizaram terbinafina, um deles por ter história familiar de tireoidite de Hashimoto e o outro por intolerância ao iodeto de potássio. O itraconazol foi usado em um paciente, que 
teve alergia ao KI (Tabela 1). Todos os pacientes obtiveram cura e queda de títulos sorológicos, que demonstra uma correlação com a resposta clínica ao tratamento (Figura 5).

\section{Discussão}

Sporothrix brasiliensis é o fungo responsável pela epidemia no estado do Rio de Janeiro. ${ }^{9}$ Tem maior perfil de virulência via pigmentação
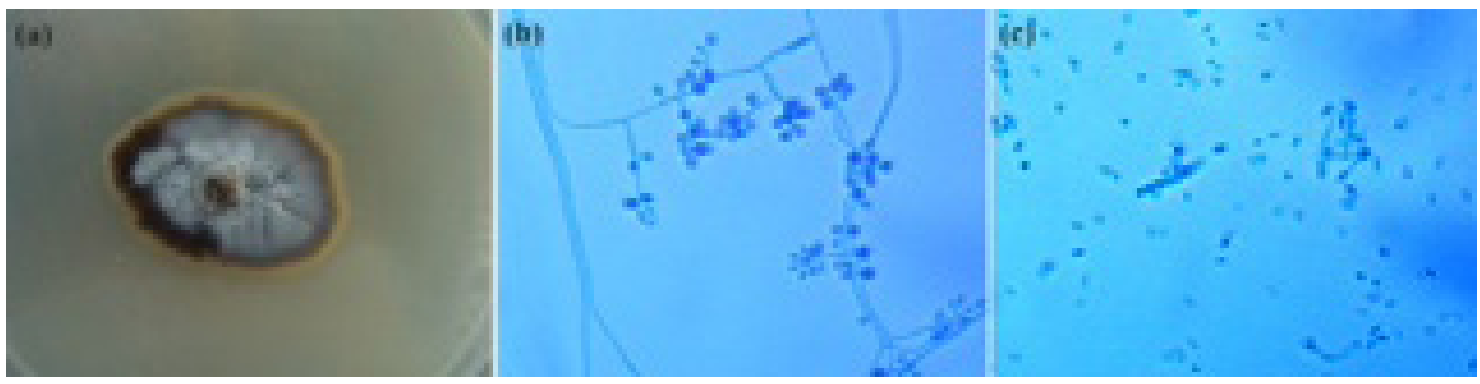

Figura 1. Sporothrix brasiliensis (a) macromorfologia a $25^{\circ} \mathrm{C}$ mostrando colônia membranosa, bege com halo enegrecido, (b) micromorfologia revela hifas hialinas, septadas, ramificadas com conidióforo perpendicular e conídios dispostos na sua extremidade em forma de "margarida" e (c) forma parasitária com elementos unicelulares alongados, formato de "charuto".

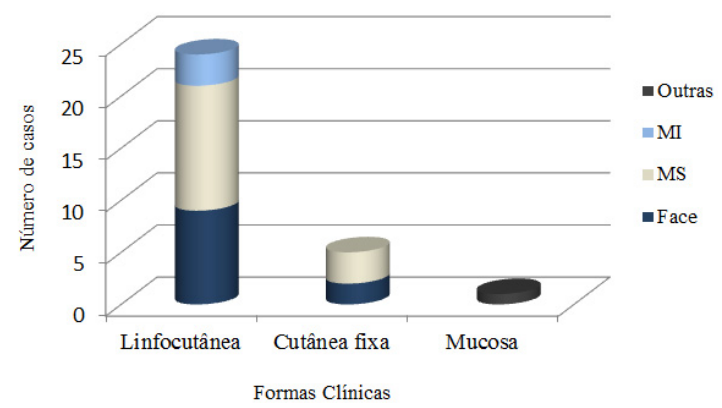

Figura 2. Distribuição das formas de contágio da esporotricose na população estudada.

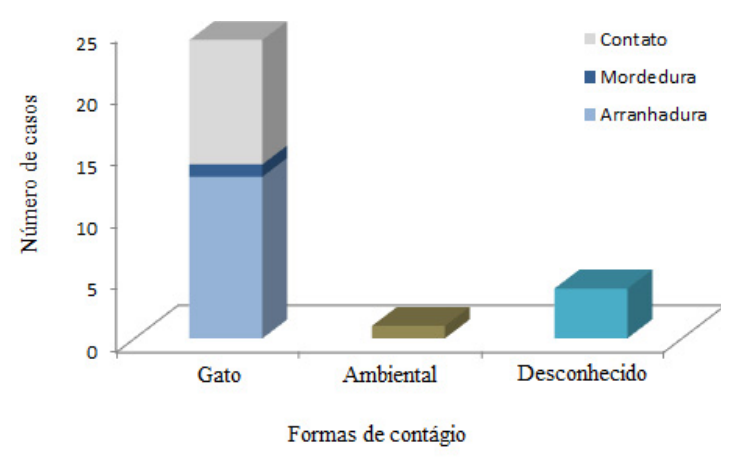

Figura 4. Distribuição das formas clínicas da esporotricose em relação à localização das lesões.
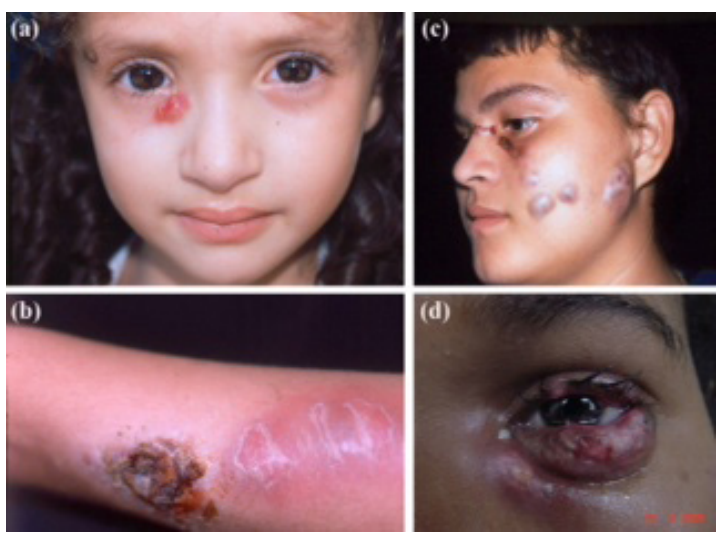

Figura 3. Formas clínicas da esporotricose: (a) cutânea fixa, (b) e (c) linfocutânea, e (d) mucosa.

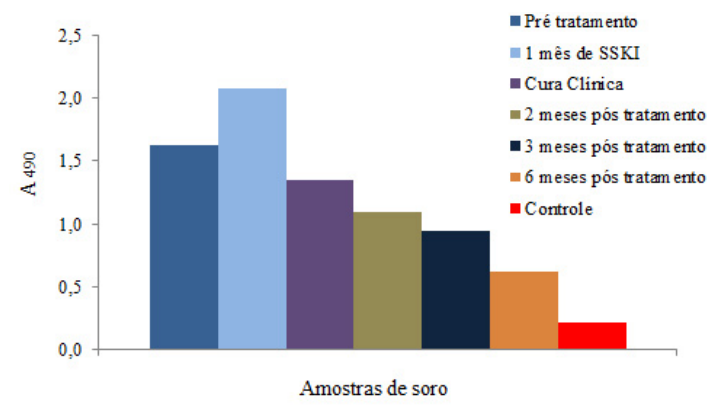

Figura 5. Avaliação sorológica de paciente da Figura 2a com análise sorológica na diluição de 1:6.400 de 6 amostras de soro antes, durante e após tratamento com SSKI. 
Tabela 1. Medicamentos utilizados para tratamento da esporotricose em crianças e adolescentes.

\begin{tabular}{|c|c|c|c|c|c|}
\hline Medicamento & SSKI & Terbinafina & Itraconazol & $\begin{array}{c}\text { Regressão } \\
\text { espontânea }\end{array}$ & $\begin{array}{c}\text { Desconhecido/ } \\
\text { Não realizado }\end{array}$ \\
\hline $\begin{array}{c}\text { Número de } \\
\text { pacientes }\end{array}$ & 25 & 2 & 1 & 1 & 10 \\
\hline $\begin{array}{c}\text { Tempo médio de } \\
\text { tratamento }\end{array}$ & 63 dias & 60 dias & 69 dias & - & - \\
\hline
\end{tabular}

Fonte: HUPE-UERJ, 1997-2010.

de conídios e produção diferenciada de exoantígenos. ${ }^{10}$ As crianças e adolescentes são alvos importantes desta epidemia pelos hábitos de conviverem e brincarem com gatos. A doença é incapacitante e, muitas vezes, afasta os indivíduos do ambiente escolar por tempo prolongado, devido ao atraso no diagnóstico e ao tempo de tratamento. Os aspectos epidemiológicos da população para esta faixa etária estão de acordo com os publicados anteriormente para a população em geral com relação à procedência, cor, sexo e formas clínicas. ${ }^{1,2,3} \mathrm{O}$ acometimento da face em mais da metade dos casos neste grupo etário é característico e se justifica pelo comportamento das crianças em brincar com felinos de forma mais próxima. O KI foi a primeira droga a ser utilizada para o tratamento da esporotricose no início do século passado e tem efetividade e segurança conhecidos. ${ }^{5}$ A solução preconizada na literatura farmacológica e em recomendações internacionais para o tratamento das formas limitadas da doença é, na verdade, concentrada (CSKI), pois é formulada com 1 $\mathrm{g}$ de $\mathrm{KI}$ em $1 \mathrm{ml}$ de água destilada, enquanto a solução saturada deveria conter 1,42 g de $\mathrm{KI}$ na forma pura (P.A.) por ml de água. ${ }^{6,8,11}$ Embora haja recomendações internacionais para o itraconazol como primeira linha de tratamento, este tem nível de evidência igual ao do KI (A-II) e a apresentação líquida da solução é mais fácil de ser administrada e aceita pelas crianças. ${ }^{6}$ Neste grupo de pacientes estudados a SSKI foi efetiva, bem tolerada e com baixos índices de eventos adversos, nenhum deles grave. A técnica sorológica, desenvolvida no Laboratório de Micologia Celular e Proteômica do Instituto de Biologia Roberto Alcântara Gomes da UERJ, é aplicada rotineiramente no Laboratório de Micologia do HUPE-UERJ. O método é realizado através de imunoensaio enzimático (ELISA) indireto, utilizando o antígeno SsCBF (obtido da parede celular de S. schenckii na fase leveduriforme). ${ }^{7,12} \mathrm{Na}$ avaliação da resposta ao tratamento foi possível comprovar a correlação clinicossorológica neste grupo de pacientes.

\section{Conclusões}

O contato com gatos constituiu a principal forma de transmissão da doença. A maioria dos pacientes apresentou a forma linfocutânea, principalmente na face e membros superiores. $\mathrm{O}$ iodeto de potássio nessa faixa etária foi efetivo e bem tolerado, sem ocorrência de eventos adversos graves e a sorologia mostrou ser ferramenta útil no diagnóstico e acompanhamento clínico dos pacientes com esporotricose.

\section{Agradecimentos}

Os autores agradecem à equipe técnica do Laboratório de Micologia HUPE-UERJ, em especial a lone Carlos da Silva e Cláudia Maria Penna Dias, pelo apoio na realização dos exames micológicos e sorológicos. Este trabalho teve apoio financeiro do Ministério da Saúde e da Fundação Carlos Chagas Filho de Amparo à Pesquisa do Estado do Rio de Janeiro (Faperj).

\section{Referências}

1. Barros MBL, Schubach TMP, Galhardo MCG. Sporotrichosis: an emergent zoonosis in Rio de Janeiro. Mem Inst Oswaldo Cruz. 2001;96(6)777-779.

2. Silva MBT, Costa MMM, Torres CCS, Esporotricose urbana: epidemia negligenciada no Rio de Janeiro, Brasil. Cad Saúde Pública. 2012;28(10):1867-1880.

3. Barros MBL, Schubach AO, Schubach TMP, An epidemic of sporotrichosis in Rio de Janeiro, Brazil: epidemiological aspects of a series of cases. Epidemiol Infect. 2008;136(9):1192-1196.

4. Bustamante B, Campos PE. Sporotrichosis 
treatment: overview and update. Curr Fungal Infect Rep. 2011;5(1):42-48.

5. Sterling JB, Heymann WR. Potassium iodide in dermatology: a $19^{\text {th }}$ century drug for the $21^{\text {st }}$ century-uses, pharmacology, adverse effects, and contraindications. J Am Acad Dermatol. 2000;43(4):691-697.

6. Kauffman CA, Bustamante B, Chapman SW, Pappas PG; Infectious Diseases Society of America. Clinical Practice Guidelines for the Management of Sporotrichosis: 2007 Update by the Infectious Diseases Society of America. IDSA Guidelines for management of Sporotrichosis. Clin Infect Dis. 2007;45(10):1255-65.

7. Bernardes-Engemann AR, Orofino RC, Miguens BP, Development of an enzyme-linked immunosorbent assay for the serodiagnosis of several clinical forms of sporotrichosis. Med Mycol. 2005;43(6):487-493.

8. Orofino-Costa R, Macedo PM, Carvalhal A, Uso do iodeto de potássio na dermatologia: considerações

Recebido: 15/05/2014.

Revisado: 02/07/2014.

Aprovado: 31/07/2014. atuais de uma droga antiga. An Bras Dermatol. 2013;88(3):401-407.

9. Rodrigues AM, Teixeira MM, Hoog GS, Phylogenetic Analysis Reveals a High Prevalence of Sporothrix brasiliensis in Feline Sporotrichosis Outbreaks. Neg Trop Dis. 2013;7(6):1-14.

10. Fernandes GF, Santos PO, Rodrigues AM, Characterization of virulence profile, protein secretion and immunogenicity of different Sporothrix schenckii sensu stricto isolates compared with S. globosa and S. brasiliensis species. Virulence. 2013;4(3):241-249.

11. Martindale. Thyroid and Antithyroid Drugs. In: Martindale: the complete drug reference. $36^{\text {th }}$ ed. USA: Pharmaceutical Press; 2009.p.2169-2170.

12. Bernardes-Engemann AR. Validação, avaliação de impacto e análise da aplicabilidade de teste de ELISA no diagnóstico da esporotricose. [Tese de doutorado]. Rio de Janeiro: UERJ; 2009

\author{
Andréa R. Bernardes-Engemann \\ Unidade Docente Assistencial de Dermatologia. Hospital \\ Universitário Pedro Ernesto. Faculdade de Ciências \\ Médicas. Universidade do Estado do Rio de Janeiro. Rio \\ de Janeiro, RJ, Brasil.
}

\section{Leila M. Lopes-Bezerra}

Instituto de Biologia Roberto Alcântara Gomes. Universidade do Estado do Rio de Janeiro. Rio de Janeiro, RJ, Brasil.

\section{Priscila M. de Macedo}

Unidade Docente Assistencial de Dermatologia. Hospital Universitário Pedro Ernesto. Faculdade de Ciências Médicas. Universidade do Estado do Rio de Janeiro. Rio de Janeiro, RJ, Brasil.

\section{Rosane Orofino-Costa}

Unidade Docente Assistencial de Dermatologia. Hospital Universitário Pedro Ernesto. Faculdade de Ciências Médicas. Universidade do Estado do Rio de Janeiro. Rio de Janeiro, RJ, Brasil. 